\title{
The Use of Virtual Manufacturing Features in forward Manufacturing Process Planning
}

\author{
Janusz Pobozniak ${ }^{\mathrm{a}}$ \\ Faculty of Mechanical Engineering, Cracow University of Technology, Al. Jana Pawła II 37, 31-864 Cracow, Poland
}

\begin{abstract}
CAPP system is the missing link between CAD/CAM system. Two types of operation of CAPP systems can be distinguished. The first one is the backward process planning, where the part is transformed from its ready state to the stock state. The second method is the forward planning, where the stock is transformed into the finished part. Backward process planning allow for the greater degree of automation, but is difficult for the acceptation among the industrial users. One of the problem in the forward process planning is the necessity to use the manufacturing features which do not occur in the finished part, they occur only in the part intermediate states. The paper propose so called virtual manufacturing features to solve this problem.
\end{abstract}

\section{CAPP system as the link between CAD/CAM}

There are several phases in the product lifecycle like concept development, cost estimation, marketing research, preliminary and final product documentation, manufacturing process planning, CNC program preparation, production start-up, etc. Computer Aided systems (CAx) with different degree of automation are used on each of these stages. The degree of automation means the degree of human assistance in the decision taking. Computer Aided Design (CAD) systems are used to develop the design documentation. The modern systems, apart from geometrical model store also the nongeometrical data like dimension tolerances, location tolerances, surface roughness, etc. After the development of the design documentation, the manufacturing process planning is started. The sequence of operation is determined during this stage. In case of machining operations, the machine tools, tools, machining fixtures, tools and machining cycles are selected. The number of set-ups necessary to machine the part is also determined. On this stage, Computer Aided Process Planning (CAPP) systems are used. The next stage is CNC machine tool programming with Computer Aided Manufacturing (CAM) systems. Usually, all works at this stage are done manually based on the already developed manufacturing processes. Because of this, CAPP systems are treated as the link between $\mathrm{CAD}$ and CAM. Automatic generation of manufacturing process plans or significant degree of human assistance allow to shorten the technical production preparation time and increase the degree of automation during CNC machine tool programming. Automation of these activities with CAPP systems offers several advantages. The first of them is the possibility to store and reuse the manufacturing knowledge. The good quality knowledge coming from experienced process planes or confirmed by laboratory research can be stored. Then it can be reused by less experienced persons, so

\footnotetext{
${ }^{\mathrm{a}}$ Corresponding author : pobozniak@mech.pk.edu.pl
} 
the newly developed processes will have the same, high quality. Additionally, such solution promotes the standardization and full use of company know-how. This aspect is especially important in view of nowadays noticed, missing engineering resources and growing complexity of manufacturing processes. The typical process plans presented in academic books contains usually only a few operations whereas the manufacturing processes in the aerospace industry usually have several dozen of operations. Their correct selection, order and contents are the factors deciding about the quality of manufacturing process plans. The other reason promoting the use of CAPP systems is the time consumption of manufacturing process planning. The typical manufacturing processes developed in the aerospace industry have several hundred pages. The use of CAPP systems significantly shortens the time needed to develop the documentation. The next point presents the use of Knowledge Based Systems (KBS) in CAPP, and forward and backward process planning technique. The goal of this point is not to make the exhaustive review of CAPP systems, as it can be found for example in $[1,2]$. but present the factors blocking the wider use of CAPP systems in industry. On this background, the virtual manufacturing features and the benefits of technology are presented.

\section{Forward and backward machining process planning}

CAPP systems are often based on expert system technology. The main elements of such solution are the knowledge database, reasoning mechanism, normative database and user interface. The knowledge databases store the appropriately represented knowledge. The most common form of knowledge representation are the production rules. The rules can test the geometrical model and PMI data. For example, they check the occurrence of particular manufacturing features like hole, pocket, dimensional and location tolerances, status of the part surfaces or part material. The values of variables used in the conditions rule can be read from the normative database, evaluated using other rules or submitted by external programs doing some calculations. The literature presents also other knowledge representations [1, 3] like for example frames or multi-level decision networks. Even if other Artificial Intelligence (AI) techniques are used like Artificial Neural Networks (ANN), Simulated Annealing (SA), Fire Fly algorithm or hybrid evolutionary approaches [1], the data about the features are still needed. The feature data are also used in heuristic procedure for process selection and operation sequencing [4]. The control mechanism is responsible for reasoning process. It check the rules (using forward or backward approach). If more than one rule is true, the rule which must be fired is selected. In more advanced solutions, the algorithm of manufacturing process planning is implemented in the control mechanism as in [3], where it is responsible for processing of manufacturing knowledge in the form of multi-level networks. For example, first the group of rules for stock selection is run. Then the group of rules for set-up determination is checked and next the group of rules for machining cycles selection is run. The user interface presents the results of reasoning and explains why the particular decisions were taken. In case of CAPP systems, this mechanism is also used to interactively execute the tasks which were not automated.

Two approaches to manufacturing process planning can be distinguished in literature [5]:

- backward process planning,

- forward process planning.

In the case of backward process planning, the part passes through a series of intermediate states, from the finished state to the stock state. The subsequent intermediate states are determined by adding the material. In order to illustrate, Figure 1 shows the rotational part along with the machining cycles. The first of these cycles is facing, the next is roughing, then groove turning, and finally hole drilling and reaming. In the backward process planning, the starting point is the finished part. The rules checking whether there are internal holes with higher accuracy are run. Since the hole meeting these requirements is found, the appropriate allowances (for final reaming and drilling) will be added to this hole. Next the rule adding the material to external rotational groove is run. Then the material corresponding to rough turning is added. This processing is continue until the shape of the stock is reached. It shall be noted that the rules refer primarily the state of the finished part. This is because all information about the design task is given in model submitted by design engineer. 


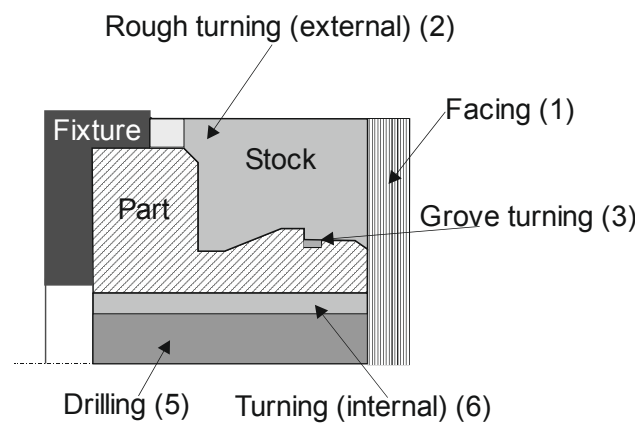

Figure 1. Machining cycles for rotational part

In the forward process planning, the subsequent machining cycles are selected, starting from the stock and ending with the finished product, which corresponds to the standard workflow of the manufacturing engineer. According to Author of this work, the main disadvantage of this method is the fact that there are manufacturing features in part intermediate states, that are not present in the part finished state. For example, the profile for external turning is not visible in the part presented on Figure 1. Such profile is the part of the profile used to create this rotational part, but it doesn't include the right planar face and profile of groove. In addition, the contour may be offset from the object when additional lathe finishing operations or grinding is used. When developing the systems for industrial users, it is not possible to use the forward design method without using such features. This is the main difficulty and the disadvantage of forward process planning method.

It shall be noted that the recognition of manufacturing features is currently one of the main obstacles in the development of CAPP systems. This is especially true in case of interacting manufacturing features $[1,6]$. If the same geometric and topological model can be decomposed into different sets of manufacturing features, this leads to the occurrence of interacting manufacturing features. An example of such features is given in Figure 2. Figure 2a) presents another version of the profile for rough turning of external surfaces for analysed part. In this case, the roughing cycle is performed first (before the facing cycle), and as previously, the pocket is not processed during this cycle. The shape of this profile for roughing cycle is quite different from the one shown earlier. Another example of the decomposition into manufacturing features is given in Figure $2 \mathrm{~b}$. In this variant, it is assumed that first machining cycle is the facing, then the contour is turned but without the pocket and without the chamfers. The pocket and chamfers are machining in the next cycles. Such strategy is usually selected in case of manually controlled lathes.

a)

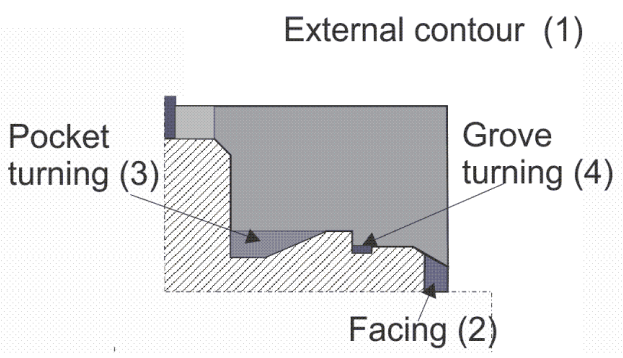

b)

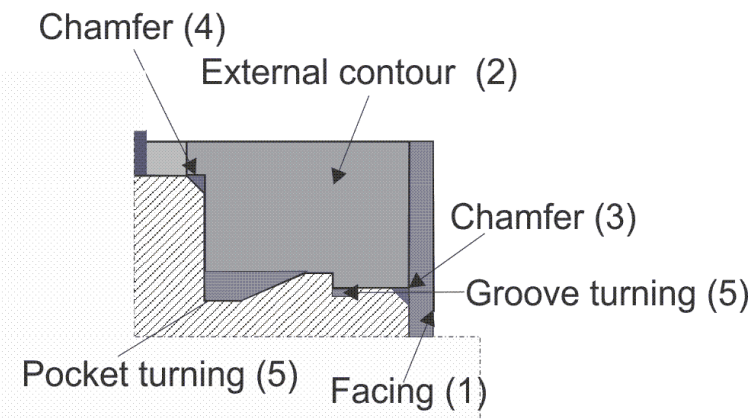

Figure 2. Interacting manufacturing features

To summary, despite many research projects on CAPP [1] and the unquestionable benefits of their usage, the provided level of automation is still unsatisfactory for commercial users. Many promising results have been obtained for CAPP systems based on backward process planning method [3]. This method could be accepted in an industrial environment in the case of complete automation. If the 
automation is partial, implementation of CAPP based on the backward method is not acceptable, especially in the case of complex manufacturing processes, consisting of several dozen operations. The paper presents the virtual manufacturing features. This solution that can be used during the development of CAPP systems based on forward process planning to use the manufacturing features which do not occur in the finished part, but occur only in the part intermediate states.

\section{Virtual manufacturing features}

Virtual manufacturing features are part of a manufacturing oriented part model.

The simplified, manufacturing oriented part model is a set of intermediate states:

$$
\mathrm{MPM}=\left\{\mathrm{IS}_{1}, \mathrm{IS}_{2}, \mathrm{IS}_{\mathrm{i}}, \mathrm{IS}_{(\mathrm{i}+1)}, \ldots, \mathrm{IS}_{\mathrm{F}}\right\}
$$

where:

MPR Manufacturing feature oriented part model

IS part intermediate state, $\mathrm{i}=1 \ldots \mathrm{F}$, for $\mathrm{i}=1$ intermediate state $\mathrm{IS}_{1}$ represents the stock, and for $\mathrm{i}=\mathrm{F}$ intermediate state $\mathrm{IS}_{\mathrm{F}}$ represents the finished state.

Each intermediate state $\mathrm{IS}_{\mathrm{i}}$ is described by a set of manufacturing features, constraints between features and operations transforming the manufacturing features into the virtual manufacturing features.

$$
\mathrm{IS}_{\mathrm{i}}=<\mathrm{F}_{\mathrm{i}}, \mathrm{CS}_{\mathrm{i}}>
$$

where:

$\mathrm{F}_{\mathrm{i}} \quad$ A set of manufacturing features in the intermediate state $\mathrm{i}$, such as holes, grooves, pockets, etc. Each feature has a semantic representation in the form of attribute sets and a full B-Rep geometric representation.

$\mathrm{CS}_{\mathrm{i}}$ Set of constraints describing the relations between features.

Manufacturing features are a basic component of manufacturing oriented part model. Manufacturing features is a specific fragment of geometric data, topological data, and non-geometric data, important from the point of view of process planning.

Virtual manufacturing feature is a result of geometric transformations on manufacturing feature using some operators. These operators can do the following changes:

- Elimination of the elements that do not meet some conditions. For example, this may involve the removal of concave elements from the milled profile, so the resultant profile is convex. This is the case for long-length profiles that include a small width concave element. The created virtual feature in the form of a convex profile is machined with a large diameter cutter, and the concave elements with the smaller diameter tool, which reduces, among others, the machining time.

- Changing the dimensions of a feature. One of the basic roughing cycles is facing. The surface for facing is always smaller than the corresponding surface of the stock. Creation of the virtual manufacturing feature extending this surface to the boundary of stock allows for proper machining of this surface.

- Changing the position of the feature relative to the geometry of the object. Such virtual manufacturing feature is always created for multi-step machining, for example divided into rough, final and super final steps. Virtual manufacturing feature is created by offsetting the profile to the distance equal to the amount of the allowance for further machining steps.

- Selection of feature elements meeting certain quality criteria. For example, operator can select surfaces with a roughness of 6.25 or less. These surfaces (elements of feature) can be subjected to final machining.

An important characteristic of a virtual manufacturing feature in comparison to normal manufacturing features is the possibility to test allowance reaming for machining. It is possible to check whether the allowance was removed at all (i.e. whether any machining cyclers related to feature were done), current dimension of the allowance and its accuracy (for example dimensional tolerance). 
The use of virtual manufacturing features is presented on Figure 3. The part is machined on a 3axis milling machine with vertical spindle. This figure shows the rules. These are typical production rules IF < Condition> Then <Actions $>$. In order to reference the manufacturing features in a condition, it is necessary to use Exists keyword, followed by the round bracket and the feature class name. The Exists function is True when there is an instance of the given feature class in the part model i.e. when specified feature exists in the part model. After the feature name, the conditions for the feature must be given, linked by logical operators. It is acceptable to use parentheses to build more complex logical expressions. For example, the rule on Figure $3 b$ has the condition $\mathbf{I} . \boldsymbol{z}=\mathbf{1}$. This condition checks whether the hole feature $\mathrm{Z}$ axis is parallel to the to the $\mathrm{Z}$ axis of the global coordinate system. The next condition, Diameter $<25$ checks if the hole diameter is less than $25 \mathrm{~mm}$. If the diameter is larger, apart from drilling, also pre-drilling is required. To check if the feature was ever machined, the value of the IS.Status (Intermediate Status.Status) is checked. If this attribute is equal to NonMachined, it means that the feature was not machined at all (the volume of material belonging to feature volume was not removed). This condition is checked by performing a geometric comparison operation.

Fig. 3c) shows the determination of a virtual manufacturing feature for surface facing. Operators acting on original manufacturing feature and creating the virtual manufacturing feature are introduced by the suffix Mod (like Modify). For example, Mod.ExtendXY operator extends the dark coloured surface in the XY plane, so the surface for facing is created. Another operator, Mod.Offset $=1$ shifts the top surface of the part by $2 \mathrm{~mm}$, so the allowance for final facing will remain. Similarly, the virtual manufacturing feature for profile milling is created (Fig. 3d). The operator Mod.Convex transform the profile for milling into the convex one. This operator removes four non-through grooves from the profile. In these examples, both surface for facing as well as concave profile are not present in the final part. These virtual manufacturing features are created, because they are needed to create the correct machining process plan. The set of such operators is defined for each manufacturing feature.

The last example (Figure 3e) shows the conditions refer to the intermediate state of the virtual manufacturing feature. There is a hole after previous drilling operation. Due to the tolerance of this hole (Hole.Tolerance.Class $=\boldsymbol{H}$ and Hole.Tolerance.Zone $<10$ ), final reaming is required. This reaming is used if the amount of reaming allowance is relatively small. In order to check the amount of remaining allowance to remove, the $\boldsymbol{I S}$ prefix with the Allowance attribute is used. If the oversize is greater than 0 and less than $1.5 \mathrm{~mm}$, this reaming is recommended. In case of a larger allowance, further drilling would be recommended. If there is a need to check the current tolerance for of the intermediate state of this hole, the condition of type IS.Tolerance.Class $=\boldsymbol{H}$ and $/$ or IS.Tolerance.Zone $>=10$ can be used. In order to check the surface roughness of this hole in the current intermediate state against the threshold value, the condition IS.Roughness. $R \boldsymbol{a}<=6.25$ is used.

After executing THEN part of any of the rules, a subtraction Boolean operation is performed to update the condition of the object. The first parameter of this operation is the volume of the part in the current intermediate state and the second parameters is the volume of material removed by the cycle. As the result, the new intermediate state is generated. So CAPP system works in the cycle:

1. Checking the rule conditions and selection of the rule to be fired from the conflict set. Other advanced processing mechanisms may also be used at this moment.

2. Transformation of the part by making the appropriate geometric Boolean operation, which results in the generation of the next intermediate state. Transformation also includes modification of PMI information, such as surface roughness or dimensional tolerance of a transformed feature.

3. Jump to step No. 1 unless the state of the finished part was reached.

The significance of the generation of intermediate states shall be noticed. These states are necessary if many $\mathrm{CNC}$ machine tools are used during manufacturing process, which is common in the aerospace industry. Intermediate states are also required for the selection of workpiece fixtures. If there are interacting manufacturing features, the decisions made by the manufacturing engineer are reflected in the generated intermediate states, which significantly reduces the number of interacting features during the next decision problems. 


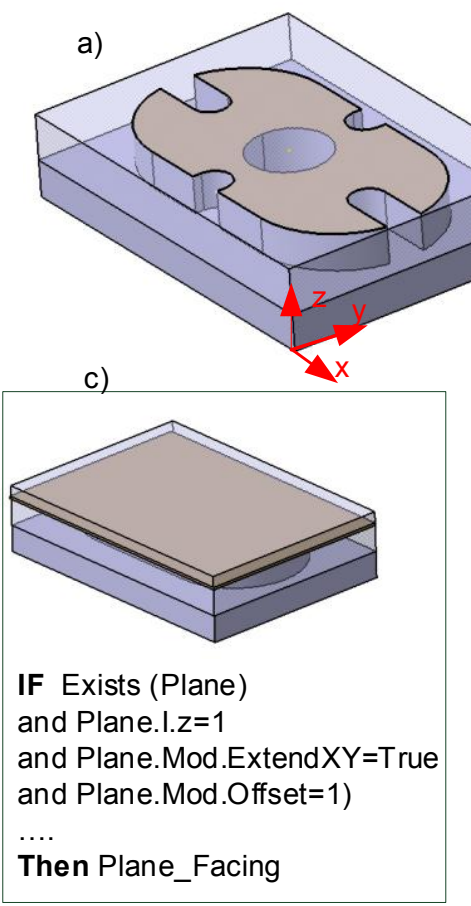

b)
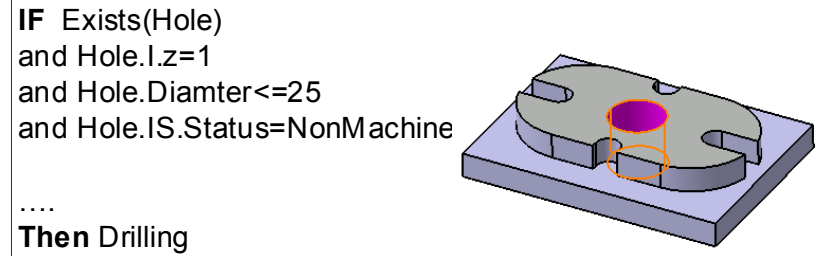

d)
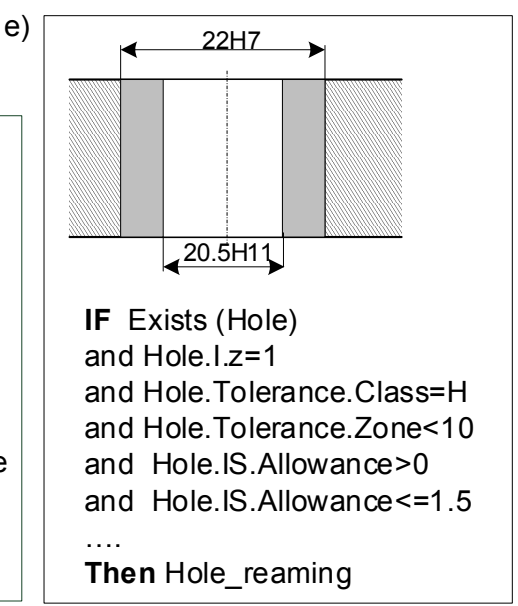

Figure 3. The use of virtual manufacturing features

\section{Summary}

The paper introduces so-called virtual technological features. These are manufacturing features that appear only in the intermediate states of the part, and are not present in the finished part. Their use allows the generation of manufacturing processes with forward planning method. The presented concept was partly verified by the development of software for recognition of manufacturing features defined in STEP-NC [2]. Further implementation work will be directed to the generation of part intermediate states based on the virtual manufacturing features, using one of the available commercial graphics kernels (OpenCascade, ACIS or C3D). The results of the work can be used during the development of CAPP systems.

\section{References}

1. Kumar Leo, S.P., State of The Art-Intense Review on Artificial Intelligence Systems Application in Process Planning and Manufacturing, In Engineering Applications of Artificial Intelligence, Volume 65, 2017

2. Chlebus E., Krot K., CAD 3D models decomposition in manufacturing processes, Archives of Civil and Mechanical Engineering, Vol. 16, 2016

3. Duda J., Pobozniak J., The Architecture of Intelligent System for CNC Machine Tool Programming, Procedia Manufacturing, Volume 11, 2017

4. Deja M.,' Siemiatkowski M., Feature-based generation of machining process plans for optimised parts manufacture, Journal of Intelligent Manufacturing, 2013

5. Chryssolouris, E.L.K.E, Manufacturing Systems: Theory and Practice, Springer-Verlag New York, 2006

6. Dipper T., Xu X., Klemm P., Defining, recognizing and representing feature interactions in a feature-based data model, Robotics and Computer-Integrated Manufacturing, Volume 27, Issue 1, 2011 